\title{
Central Memory Immune Cell
}

National Cancer Institute

\section{Source}

National Cancer Institute. Central Memory Immune Cell. NCI Thesaurus. Code C122730.

A population of long-lived antigen-specific immune cells, usually T-lymphocytes

expressing CD4, L-selectin and CCR7, that are maintained by the immune system. Upon

subsequent exposure to their target antigen, these cells rapidly proliferate and

differentiate into effector cells. 